PAPER

\title{
Atrial fibrillation as a predictive factor for severe stroke and early death in 15831 patients with acute ischaemic stroke
}

\author{
K Kimura, K Minematsu, T Yamaguchi, for the Japan Multicenter Stroke Investigators' \\ Collaboration (J-MUSIC)
}

J Neurol Neurosurg Psychiatry 2005;76:679-683. doi: 10.1136/jnnp.2004.048827

See end of article for authors' affiliations

....................

Correspondence to: Dr K Kimura, Cerebrovascular Division, Department of Medicine, Kawasaki Medical School, 557 Matsushima, Kurashiki City, Ókayama 701-0192, Japan; kimurak@med.

kawasaki-m.ac.jp

Received 29 June 2004 Revised version received 8 September 2004 Accepted

8 September 2004

\begin{abstract}
Background: Atrial fibrillation (AF) is a common arrhythmia and a major risk factor for stroke.
Aims: To assess whether AF in patients with acute ischaemic stroke is associated with severe stroke and early death.

Materials/methods: Patients with acute ischaemic stroke (15 831) who were registered in the Japan Multicenter Stroke Investigators' Collaboration registry were analysed. The AF group comprised 3335 (21.1\%) patients (median age, 75 years) and the non-AF group comprised 12496 (78.9\%) patients (median age, 70 years). The association between AF and severe stroke and early death was investigated by means of multivariate logistic regression analysis.

Results: The admission National Institutes of Health Stroke Scale (NIHSS) score of the AF group was higher than that of the non-AF group (median, 12 v 5; $p<0.0001$ ). Multivariate logistic regression analyses found that female sex, advanced age, AF, and a history of stroke were independent factors associated with severe stroke (NIHSS score, $\geqslant 11$ ). The mortality rate within 28 days after admission was $11.3 \%$ in the AF group and $3.4 \%$ in the non-AF group $(p<0.0001)$. Multivariate logistic regression analyses identified older age, AF, and NIHSS score at admission as independent factors associated with early death.

Conclusion: AF was a predictive factor for severe stroke and early death in acute ischaemic stroke. Careful cardiac evaluation and appropriate treatment are needed to improve outcome in patients with acute stroke and $\mathrm{AF}$.
\end{abstract}

A trial fibrillation (AF) is a common arrhythmia that is associated with a high risk of stroke, particularly cardioembolic stroke. ${ }^{1}$ AF carries an annual risk of thromboembolic complications of $3-6 \%$, which is five to seven times greater than that of controls with sinus rhythm..$^{2-4}$ AF is present in $15-21 \%$ of patients affected by stroke. ${ }^{5-11}$ In a clinical study in Hokkaido, a northern part of Japan, the incidence of ischaemic events in 20000 patients with AF, who were followed up in cardiovascular clinics, was $4.6 \%$ during a 1.7 year follow up period. ${ }^{12}$

Patients with AF have an increased risk of major, disabling stroke, often caused by large infarctions in the middle cerebral artery territory. ${ }^{13-15}$ Some studies demonstrated that AF was associated with an increased risk of death in the first four weeks after stroke. ${ }^{516}$ This association was explained by several factors, including the advanced age in stroke patients with AF, large infarction, severe neurological deficits, and poor functional outcomes. However, it is not clear whether AF itself is associated with early death in patients with acute ischaemic stroke.

We conducted a nationwide prospective survey from May 1999 to April 2000 in Japan (Japan Multicenter Stroke Investigators' Collaboration; J-MUSIC) to clarify the current status of stroke management and outcome. In total, 16922 patients were registered to J-MUSIC. ${ }^{17}{ }^{18}$ We performed retrospective and secondary analyses to assess whether $\mathrm{AF}$ was associated with severe stroke and early death in patients with acute ischaemic stroke, using data from the large sample of hospitalised patients registered in J-MUSIC.

\section{MATERIALS AND METHODS}

We conducted a multicentre prospective hospital based registration study (J-MUSIC) from May 1999 to April 2000 in which 156 hospitals participated. ${ }^{17}{ }^{18}$ In total, 16922 consecutive patients with acute ischaemic stroke and transient ischaemic attack (TIA) within seven days of onset were registered in our study. ${ }^{17}$ We excluded 1091 TIA patients, and thus enrolled 15831 patients with acute stroke into our present study. First, we divided patients into two groups based on the presence of $\mathrm{AF}$ : the AF group and the non-AF group. We compared the baseline and clinical characteristics and outcome between these two groups. Second, we used multivariate logistic regression analyses to investigate the association between $\mathrm{AF}$ and severe stroke, and early death. Early death was defined as death within 28 days after admission. Informed consent was obtained from all patients participating in our study.

For all patients, the following data from the common stroke protocol were assessed: (1) age and sex; (2) past history of stroke; (3) National Institutes of Health Stroke Scale (NIHSS) score on admission; (4) time from stroke onset to arrival at hospital; (5) cardiovascular risk factors (hypertension, diabetes mellitus, hyperlipidaemia, AF, and current smoking); (6) treatment, including thrombolytic treatment, heparin, and aspirin; (7) death within 28 days after admission; (8) hospital discharge status; and (9) residence after hospital discharge.

$\mathrm{AF}$ included both paroxysmal $\mathrm{AF}$ and persistent $\mathrm{AF}$ identified by electrocardiography (ECG) and/or 24 hour ECG monitoring during admission. Patients with a history of paroxysmal AF confirmed by ECG were also classified as patients with AF. The hospital discharge status was evaluated by using the modified Rankin scale score $(\mathrm{mRS})^{19}$ and death. Good outcome was defined as mRS scores of 0,1 , and 2. Residence after hospital discharge was classified into two groups: the patient's own home and an institution (including nursing home and another hospital for rehabilitation and medical management).

Abbreviations: AF, atrial fibrillation; $\mathrm{Cl}$, confidence interval; $\mathrm{ECG}$, electrocardiography; J-MUSIC, Japan Multicenter Stroke Investigators' Collaboration; mRS, modified Rankin scale; NIHSS, National Institutes of Health Stroke Scale; OR, odds ratio; TIA, transient ischaemic attack 
Table 1 Baseline characteristics of patients with and without $A F$

\begin{tabular}{|c|c|c|c|c|}
\hline Characteristics & $\begin{array}{l}\text { Total } \\
N=15831\end{array}$ & $\begin{array}{l}\text { With AF } \\
N=3335\end{array}$ & $\begin{array}{l}\text { Without AF } \\
N=12496\end{array}$ & p Value \\
\hline Mean (SD; median) age (years) & $70.7(11.5 ; 71)$ & $74.5(9.8 ; 75)$ & $69.6(11.7 ; 70)$ & $<0.0001$ \\
\hline$<45(\%)$ & 1.9 & 0.4 & 2.3 & \\
\hline $45-54(\%)$ & 7.6 & 2.8 & 8.9 & \\
\hline $55-64(\%)$ & 19.5 & 14.4 & 20.8 & \\
\hline $65-74(\%)$ & 33.2 & 31.8 & 33.5 & \\
\hline $75-84(\%)$ & 28.2 & 36.0 & 26.1 & \\
\hline$>84(\%)$ & 9.8 & 14.6 & 8.5 & \\
\hline Female (\%) & 38.7 & 46.5 & 38.0 & 0.0003 \\
\hline History of stroke (\%) & 34.2 & 35.6 & 30.1 & $<0.0001$ \\
\hline \multicolumn{5}{|l|}{ Risk factors } \\
\hline Hypertension (\%) & 61.2 & 48.7 & 64.5 & $<0.0001$ \\
\hline Diabetes mellitus (\%) & 24.7 & 16.9 & 26.8 & $<0.0001$ \\
\hline Hyperlipidaemia (\%) & 16.6 & 9.5 & 18.5 & $<0.0001$ \\
\hline Smoking (\%) & 17.3 & 10.2 & 19.2 & $<0.0001$ \\
\hline NIHSS score (mean, SD, median) & $8.3,7.9,5$ & $13.7,9.7,12$ & $6.9,6.7,5$ & $<0.0001$ \\
\hline $0-6(\%)$ & 57.4 & 31.3 & 64.4 & \\
\hline $7-10(\%)$ & 15.4 & 12.7 & 16.2 & \\
\hline $11-15(\%)$ & 9.9 & 15.3 & 8.5 & \\
\hline $16-22(\%)$ & 9.6 & 21.0 & 6.5 & \\
\hline$\geqslant 23(\%)$ & 7.7 & 19.7 & 4.5 & \\
\hline
\end{tabular}

\section{Statistical analysis}

Statistical analyses were performed with a commercially available software package (Stat-view, version 4.5; SAS Institute, Cary, North Carolina, USA). The Mann-Whitney $\mathrm{U}$ test was used to detect differences in age and NIHSS scores
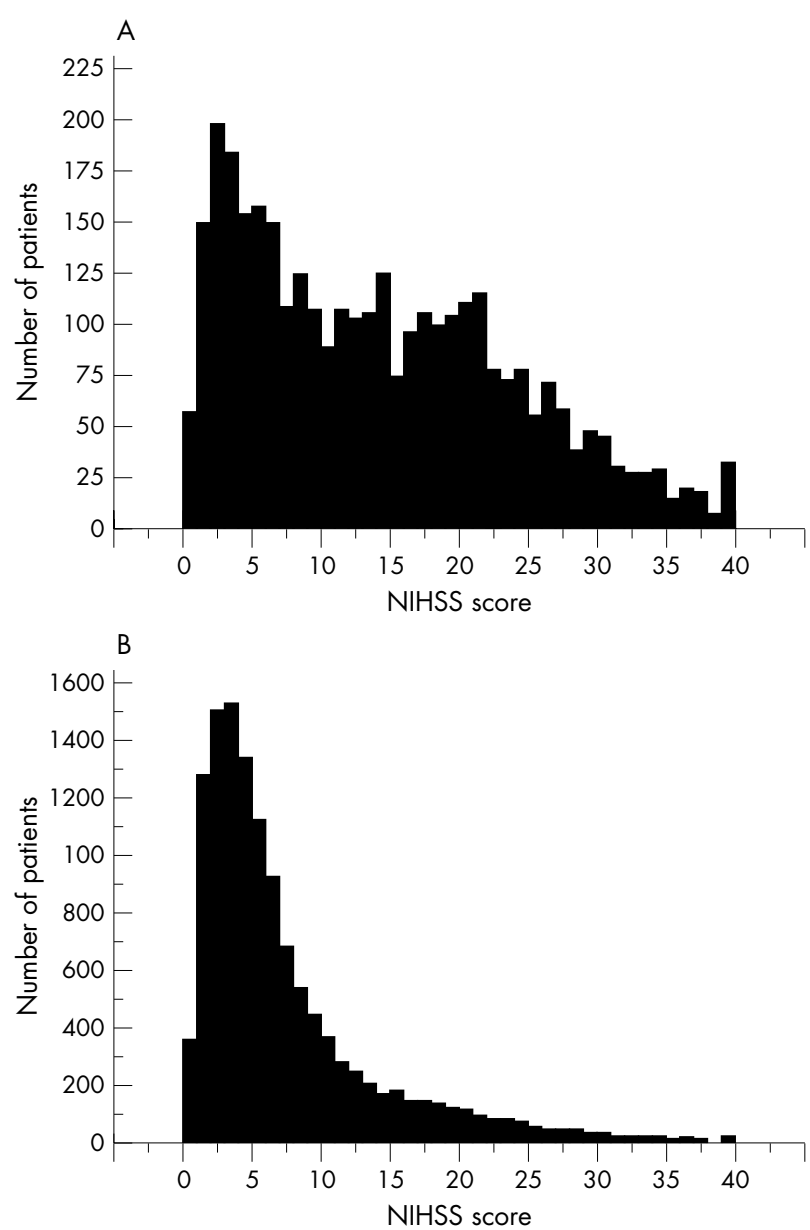

Figure 1 Distribution of National Institutes of Health Stroke Scale (NIHSS) scores for (A) patients with atrial fibrillation (AF) and (B) patients without AF. among the groups. All other differences were assessed using the $\chi^{2}$ test. We divided the patients into five groups based on stroke severity (NIHSS score: $\leqslant 6,7-10,11-15,16-22$, and $\geqslant 23$ ) according to those in the TOAST study..$^{20}$ Multivariate logistic regression models were used to identify factors associated with mild stroke (NIHSS scores, $\leqslant 6$ ) and severe stroke (NIHSS scores, $\geqslant 11$ ) at admission. Furthermore, multivariate logistic regression models were applied to identify factors associated with early death after admission. Next, we examined the early death rate in patients with and without AF by five groups based on stroke severity. According to the results, we divided patients into subgroups and applied multivariate logistic regression models to identify factors associated with early death for each group. Variables $(\mathrm{p}<0.20)$ associated with stroke severity and early death in the univariate analysis were selected to be evaluated in the multivariate logistic regression analyses. Differences were considered significant at the level of $\mathrm{p}<0.05$.

\section{RESULTS}

The mean age of all patients (6130 women and 9701 men) was 70.7 (SD, 11.5) years (median, 71; range, 18-107). The AF group comprised 3335 patients $(21.1 \%)$ and the non-AF group comprised 12496 patients (78.9\%). Table l shows the baseline features of the patients with and without AF.

\section{Severity of stroke (NIHSS on admission)}

The mean (SD) and median NIHSS scores for all patients were 8.3 (7.9) and 5. The scores were significantly higher in the AF group (mean, 13.7; SD, 9.7; median, 12) than in the non-AF group (mean, 6.9; SD, 6.7; median, 5) (p<0.0001; fig 1). AF was seen in $11.5 \%$ of patients with NIHSS scores of $0-6,17.4 \%$ of patients with scores of $7-10,32.5 \%$ of patients with scores of $11-15,46.3 \%$ of patients with scores of 16-22, and $54.0 \%$ of patients with scores $\geqslant 23(p<0.0001)$. Thus, the frequency of AF rose steeply as NIHSS scores increased. The mean (SD) and median NIHSS scores were 7.4 (8.2) and 4 in patients aged less than 45 years, 5.8 (6.2) and 4 in patients aged 45-54 years, 6.6 (6.9) and 4 in patients aged 55-64 years, $7.8(7.6)$ and 5 in patients aged $65-74$ years, 9.6 (8.3) and 7 in patients aged 75-84 years, and $12.3(9.0)$ and 10 in patients aged 85 years or older $(p<0.0001)$. Multivariate logistic regression analysis identified female sex, increased age, AF, and history of stroke as independent factors associated with severe stroke (table 2). 
Table 2 Multivariate logistic regression analysis models for probability of mild and severe neurological deficits

\begin{tabular}{|c|c|c|c|c|c|c|}
\hline \multirow[b]{2}{*}{ Variable } & \multicolumn{3}{|c|}{ NIHSS score $\leqslant 6$} & \multicolumn{3}{|c|}{ NIHSS score $\geqslant 11$} \\
\hline & OR & $95 \% \mathrm{Cl}$ & $\mathrm{p}$ Value & OR & $95 \% \mathrm{Cl}$ & $\mathrm{p}$ Value \\
\hline Female sex & 0.85 & 0.789 to 0.914 & $<0.0001$ & 1.25 & 1.150 to 1.356 & $<0.0001$ \\
\hline Age difference of 1 year & 0.97 & 0.970 to 0.976 & $<0.0001$ & 1.03 & 1.025 to 1.033 & $<0.0001$ \\
\hline Hypertension & 1.15 & 1.068 to 1.230 & 0.0002 & 0.79 & 0.728 to 0.853 & $<0.0001$ \\
\hline Diabetes mellitus & 0.92 & 0.846 to 0.992 & 0.031 & 0.92 & 0.836 to 1.008 & 0.0728 \\
\hline Hypercholesterolaemia & 1.22 & 1.109 to 1.338 & $<0.0001$ & 0.77 & 0.687 to 0.862 & $<0.0001$ \\
\hline Atrial fibrillation & 0.29 & 0.268 to 0.318 & $<0.0001$ & 4.43 & 4.067 to 4.828 & $<0.0001$ \\
\hline Current smoking & 1.09 & 0.985 to 1.195 & 0.097 & 0.83 & 0.735 to 0.929 & 0.001 \\
\hline History of stroke & 0.72 & 0.667 to 0.772 & $<0.0001$ & 1.32 & 1.216 to 1.433 & $<0.0001$ \\
\hline
\end{tabular}

$\mathrm{Cl}$, confidence interval; NIHSS, National Institutes of Health Stroke Scale; OR, odds ratio.

Time between stroke onset and hospital arrival

Thirty five per cent of patients were admitted within three hours of stroke onset. The cumulative frequency was $48.1 \%$ within six hours, $58.4 \%$ within 12 hours, $71.7 \%$ within 24 hours, $83.6 \%$ within 48 hours, and $91.0 \%$ within 72 hours. Both the frequency of AF and the NIHSS score at admission were higher in patients admitted within 24 hours of onset than in those admitted after 24 hours (frequency of AF: 85.6\% v 68.1\%; $\mathrm{p}<0.0001$; NIHSS score: mean, 9.5; SD, 8.5; median $6 v$ mean, 5.5; SD, 5.4; median, $4 ; \mathrm{p}<0.0001)$.

\section{Treatment within 12 hours of stroke onset}

When we define thrombolytic treatment as intravenous tissue plasminogen activator or intra-arterial urokinase or tissue plasminogen activator, $7.3 \%$ of the AF group and $1.3 \%$ of the non-AF group received thrombolytic treatment during the superacute phase of their stroke.

\section{Treatment within seven days of stroke onset}

Heparin was administered to $38.1 \%$ of the AF group and to $10.6 \%$ of the non-AF group $(\mathrm{p}<0.0001)$, whereas aspirin was given to $7.0 \%$ of the AF group and to $10.5 \%$ of the nonAF group $(\mathrm{p}<0.0001)$.

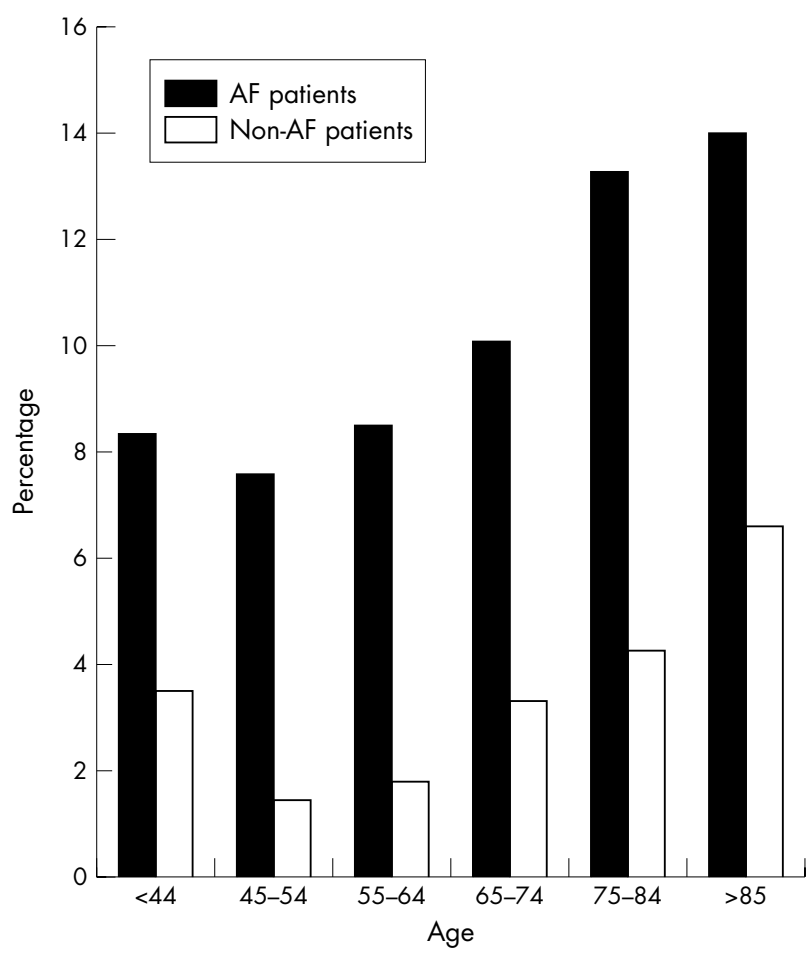

Figure 2 Death within 28 days of admission and age for patients with atrial fibrillation (AF) and non-AF patients.

\section{Length of hospital stay}

The mean (SD) length of hospital stay for all patients was 35.3 (34.1) days (median, 25; range, 1-429). The mean (SD) length of hospital stay for the AF group was 40.5 (37.8) days (median, 29; range, 0-374) and for the non-AF group 34.0 (32.9) days (median, 24; range, $0-429)(\mathrm{p}<0.0001)$.

\section{Death within 28 days of admission}

A total of 804 patients $(5.1 \%)$ died within 28 days of admission. Early death was more frequent in patients admitted within 24 hours of onset than in those admitted after 24 hours $(6.5 \%$ v $1.5 \%$; p $<0.0001)$. Their mean (SD) survival time was 8.3 (7.5) days (median, 5; range, $0-28$ ). The mortality rate was $11.3 \%$ in the AF group and $3.4 \%$ in the non-AF group $(p<0.0001)$. The mortality rate was higher in the patients with AF than in those without AF for each age group ( $p<0.0001$ for each group; fig 2 ). Univariate analysis showed that older age, higher NIHSS score, presence of AF, history of stroke, thrombolytic treatment, and the use of heparin were higher in the early death group (table 3). Multivariate logistic regression analysis identified age, AF, and NIHSS score at admission as independent factors associated with early death (table 3 ). Figure 3 shows the death rate in patients with and without AF stratified by NIHSS score group. In patients with NIHSS scores of 0-6 and $7-10$, the death rate of patients with AF was higher than that of those without ( $p=0.0001$ and $p=0.0123$, respectively). However, no significant differences were seen in those with NIHSS scores of 11-15, 16-22, and $>23$. Therefore, we

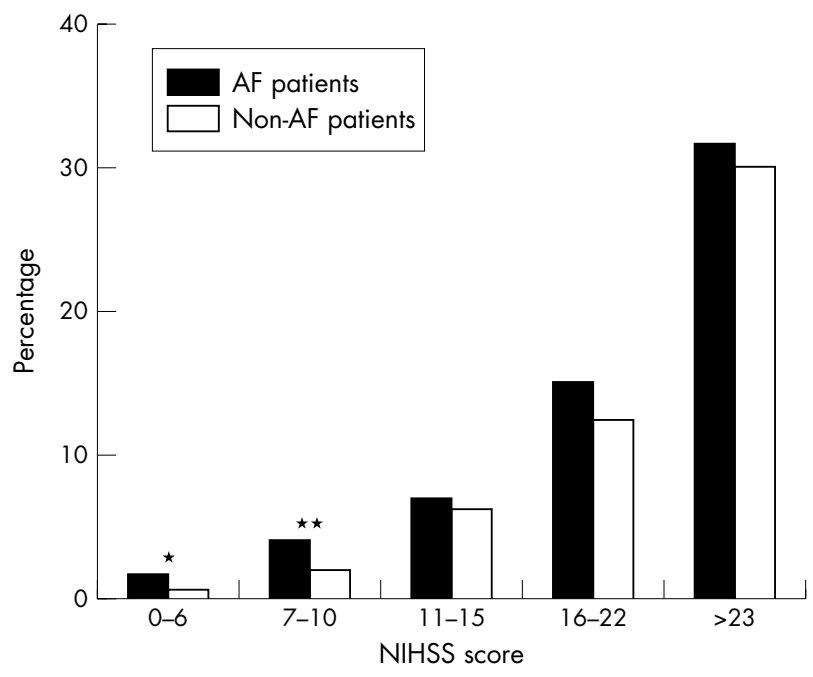

Figure 3 Death in acute stroke in patients with and without atrial fibrillation (AF), according to the severity of stroke at admission. The death rate of patients with $\mathrm{AF}$ is higher than that of the non-AF patients in the subgroup of patients with mild stroke. 
Table 3 Multivariate logistic regression analysis models for probability of early death

\begin{tabular}{|c|c|c|c|c|c|c|}
\hline \multirow[b]{3}{*}{ Variable } & \multicolumn{3}{|c|}{ Univariate analysis } & \multicolumn{3}{|c|}{ Multivariate logistic regression analysis } \\
\hline & \multicolumn{3}{|l|}{ Early death } & \multirow[b]{2}{*}{ OR } & \multirow[b]{2}{*}{$95 \% \mathrm{Cl}$} & \multirow[b]{2}{*}{ p Value } \\
\hline & Yes $(n=804)$ & No $(n=15004)$ & p Value & & & \\
\hline Female & $45.9 \%$ & $38.4 \%$ & $<0.0001$ & 1.00 & 0.841 to 1.178 & 0.957 \\
\hline Age in years (mean, SD, median) & $74.8,10.8,75$ & $70.4,11.5,71$ & $<0.0001$ & $1.01^{*}$ & 1.005 to 1.020 & 0.002 \\
\hline Hypertension & $52.6 \%$ & $61.7 \%$ & $<0.0001$ & 0.95 & 0.809 to 1.119 & 0.957 \\
\hline Diabetes mellitus & $20.9 \%$ & $24.9 \%$ & 0.011 & 1.11 & 0.912 to 1.354 & 0.297 \\
\hline Hypercholesterolaemia & $8.6 \%$ & $17.7 \%$ & $<0.0001$ & 0.75 & 0.570 to 0.990 & 0.042 \\
\hline Atrial fibrillation & $47.8 \%$ & $19.6 \%$ & $<0.0001$ & 1.27 & 1.061 to 1.510 & 0.009 \\
\hline Current smoking & $10.2 \%$ & $17.7 \%$ & $<0.0001$ & 0.88 & 0.680 to 1.148 & 0.353 \\
\hline History of stroke & $35.0 \%$ & $31.1 \%$ & 0.021 & - & - & - \\
\hline Thrombolytic treatment & $17.0 \%$ & $4.8 \%$ & $<0.0001$ & 1.34 & 1.007 to 1.795 & 0.045 \\
\hline Aspirin & $1.9 \%$ & $10.1 \%$ & $<0.0001$ & 0.30 & 0.175 to 0.503 & $<0.0001$ \\
\hline Heparin & $23.5 \%$ & $16.0 \%$ & $<0.0001$ & 0.91 & 0.750 to 1.108 & 0.352 \\
\hline $\begin{array}{l}\text { NIHSS score on admission (mean, SD, } \\
\text { median) }\end{array}$ & $21.5,9.7,22$ & $7.6,7.2,5$ & $<0.0001$ & $1.15 \dagger$ & 1.137 to 1.155 & $<0.0001$ \\
\hline
\end{tabular}

${ }^{*}$ Analysis by difference of 1 year; tanalysis by difference of 1 point.

$\mathrm{Cl}$, confidence interval; NIHSS, National Institutes of Health Stroke Scale; OR, odds ratio.

performed multivariate logistic regression analysis for probability of early death in patients with NIHSS scores $<11$ and $\geqslant 11$. AF was an independent factor associated with early death in patients with NIHSS scores $<11$, but not in those with NIHSS scores $\geqslant 11$ (table 4 ).

\section{Hospital discharge status}

The proportions of patients with each mRS score at discharge in the AF and non-AF groups were as follows: $9.4 \%$ and $15.7 \%$ for score $0,18.4 \%$ and $33.2 \%$ for score $1,11.6 \%$ and $14.8 \%$ for score $2,7.7 \%$ and $9.1 \%$ for score $3,16.9 \%$ and $14.7 \%$ for score $4,19.6 \%$ and $7.6 \%$ for score 5 , and $16.4 \%$ and $5.0 \%$ for death, respectively. Good outcome was observed in $39.4 \%$ of patients with AF and in $63.7 \%$ of patients without AF, respectively ( $p<0.0001$ ). Multivariate logistic regression analysis showed that older age (odds ratio (OR), 1.03; 95\% confidence interval (CI), 1.02 to 1.04), the presence of $\mathrm{AF}$ (OR, 1.3; 95\% CI, 1.14 to 1.55), diabetes mellitus (OR, 1.2; 95\% CI, 1.02 to 1.44 ), and NIHSS score at admission (OR, 1.1; 95\% CI, 1.14 to 1.15) were independent factors associated with death.

\section{Residence after hospital discharge}

Sixty two per cent of all patients were discharged home and $38 \%$ were sent to an institution. Of the patients without $\mathrm{AF}$, $66.4 \%$ returned to their own homes, whereas only $45.1 \%$ of patients with AF returned home $(\mathrm{p}<0.0001)$.

\section{DISCUSSION}

Our study showed that AF was clearly associated with an increased risk of severe neurological deficits. The fact that patients with AF have more severe stroke than those without
AF supports the hypothesis that the pathogenetic mechanism of stroke may be different. First, strokes in patients with AF may chiefly be cardioembolic, which causes a sudden occlusion of large cerebral arteries without sufficient collateral blood flow, resulting in more severe strokes. ${ }^{12-14}$ Several studies have reported that stroke patients with AF more often have large cortical infarcts on computed tomography, and less frequently have lacunar infarction compared with patients without AF. ${ }^{51015}$ Second, a previous study found a significant reduction in hemispheric cerebral blood flow in patients with AF compared with those with sinus rhythm. ${ }^{21}$ The effect of the decreased hemispheric cerebral blood flow may contribute to the development of large infarcts and neurological severity in patients with AF.

Our present study showed that older age and higher NIHSS score at admission were independent factors associated with early death, results that agreed with previous reports. ${ }^{20} 223$ Furthermore, we identified AF as an independent factor for early death, in particular, for patients with NIHSS scores 010. Previous studies have reported that AF was associated with an increased risk of early death. ${ }^{5916}$ This was explained by its association with severe neurological deficits and the older age of patients with AF. However, not all investigators agreed that $\mathrm{AF}$ itself increased the risk of death. ${ }^{8425}$ In our present study, after adjustment for age and NIHSS score using multivariate logistic analysis, AF was identified as an independent risk factor for early death, which was compatible with the results of the Oxfordshire community stroke project. ${ }^{10}$

Heart diseases are more frequent in patients with AF than in those without. ${ }^{26}{ }^{27}$ Some studies have suggested that

Table 4 Multivariate logistic regression analysis models for probability of early death in patients with NIHSS $<11$ and $\geqslant 11$

\begin{tabular}{|c|c|c|c|c|c|c|}
\hline \multirow[b]{2}{*}{ Variable } & \multicolumn{3}{|c|}{ NIHSS score < 11} & \multicolumn{3}{|c|}{ NIHSS score $\geqslant 11$} \\
\hline & OR & $95 \% \mathrm{Cl}$ & p Value & OR & $95 \% \mathrm{Cl}$ & p Value \\
\hline Age (years) & $1.02^{*}$ & 1.004 to 1.041 & 0.014 & $1.01^{*}$ & 0.998 to 1.014 & 0.141 \\
\hline Hypertension & - & - & - & 0.96 & 0.805 to 1.145 & 0.650 \\
\hline Hypercholesterolaemia & 0.59 & 0.323 to 1.082 & 0.088 & 0.85 & 0.624 to 1.153 & 0.293 \\
\hline Atrial fibrillation & 1.88 & 1.210 to 2.924 & 0.005 & 1.11 & 0.928 to 1.338 & 0.248 \\
\hline Current smoking & - & - & - & 0.88 & 0.657 to 1.181 & 0.397 \\
\hline Thrombolytic treatment & 3.58 & 1.474 to 8.677 & 0.005 & 1.18 & 0.885 to 1.584 & 0.255 \\
\hline Aspirin & 0.52 & 0.242 to 1.127 & 0.0976 & 0.22 & 0.105 to 0.440 & $<0.0001$ \\
\hline Heparin & 1.26 & 0.776 to 2.034 & 0.3523 & 0.80 & 0.653 to 0.986 & 0.037 \\
\hline NIHSS score on admission & $1.23+$ & 1.153 to 1.319 & $<0.0001$ & $1.11 \dagger$ & 1.098 to 1.124 & $<0.0001$ \\
\hline
\end{tabular}

${ }^{*}$ Analysis by difference of 1 year; tanalysis by difference of 1 point.

$\mathrm{Cl}$, confidence interval; NIHSS, National Institutes of Health Stroke Scale; OR, odds ratio. 
cardiac causes of death in the acute phase of stroke predominate in patients with AF compared with those without AF. ${ }^{28} 29$ Tomita et al reported that $77 \%$ of 2677 patients with AF had heart diseases such as hypertensive heart disease, ischaemic heart disease, valvular heart disease, sick sinus syndrome, and cardiomyopathy. ${ }^{12}$ In our study, the mortality rate in patients with NIHSS scores of $<11$ was higher in patients with AF than in non-AF patients. Death in such patients may be caused by stroke complications, such as heart diseases and pneumonia, rather than stroke itself. Careful cardiac evaluation and treatment are needed in acute stroke patients with AF even if patients' neurological deficits are mild. Furthermore, Lin et al reported that the one year survival rate was lower in patients with AF compared with those without AF. ${ }^{30}$ Secondary prevention of embolic events is one of the most important issues for patients with AF.

Our present study has some limitations. First, the definition of AF in our study included both chronic AF and paroxysmal AF. The frequency of paroxysmal AF is reported to be about half that of chronic AF in Japan. ${ }^{12}$ Thus, the presence of paroxysmal AF may not have been fully recognised in our present study. Second, we did not assess stroke recurrence and the cause of death, such as severe stroke, stroke recurrence, heart failure, renal failure, pneumonia, and pulmonary embolism. Some studies reported no difference in the frequency of stroke recurrence between acute stroke patients with and without AF, ${ }^{22} 29$ whereas other studies disagreed with these findings. ${ }^{31} 32$ Further studies are needed to investigate stroke recurrence and the cause of death related to AF. Third, Saxena et al reported that AF was a high risk carried early death, which could be explained by older age and large infarcts. ${ }^{22}$ Unfortunately, we did not examine the neuroimaging findings, such as computed tomography and magnetic resonance imaging. Fourth, the NIHSS score at admission may be affected by some previous neurological deficits, particularly in patients with a history of stroke. The severity of stroke at admission in patients with AF might be estimated to be severe compared with those without AF because patients with AF more often had a history of stroke than those without AF. Finally, early aspirin use is of benefit in acute ischaemic stroke. ${ }^{33}$ However, few patients in our study were treated with aspirin during the acute phase of stroke. The death rate in our present study might have been lower if aspirin had been used more frequently in patients in the acute phase of stroke.

In conclusion, our study showed that $\mathrm{AF}$ was a predictive factor for both severe stroke and early death in acute ischaemic stroke. Therefore, careful cardiac evaluation and appropriate treatment are needed to improve the outcome in patients with acute stroke and AF.

\section{ACKNOWLEDGEMENTS}

This study was supported by Health Sciences Research Grants (19982000) from the Ministry of Health, Labour and Welfare, Japan.

\section{Authors' affiliations}

K Kimura, K Minematsu, T Yamaguchi, Cerebrovascular Division, Department of Medicine, National Cardiovascular Centre, Kawasaki Medical School, 557 Matsushima, Kurashiki City, Okayama 701-0192, Japan

Competing interests: none declared

\section{REFERENCES}

1 Cerebral Embolism Task Force. Cardiogenic brain embolism. Arch Neurol 1986;43:71-84.
2 Wolf PA, Dawber TR, Thomas HE Jr, et al. Epidemiologic assessment of chronic atrial fibrillation and risk of stroke: the Framingham study. Neurology 1978;28:973-7.

3 Petersen P. Thromboembolic complications in atrial fibrillation. Stroke 1990;21:4-13.

4 Anonymous. Risk factors for stroke and efficacy of antithrombotic therapy in atrial fibrillation. Analysis of pooled data from five randomized controlled trials. Arch Intern Med 1994;154:1449-57.

5 Britton M, Gustafsson C. Non-rheumatic atrial fibrillation as a risk factor for stroke. Stroke 1985;16:182-8.

6 Wolf PA, Abbott RD, Kannel WB. Atrial fibrillation: a major contributor to stroke in the elderly. The Framingham study. Arch Intern Med 1987; 147:1561-4

7 Candelise L, Pinardi G, Morabito A. Mortality in acute stroke with atrial fibrillation. The Italian acute stroke study group. Stroke 1991;22:169-74.

8 Friedman PJ. Atrial fibrillation after stroke in the elderly. Stroke $1991 ; 22: 209-14$.

9 Broderick JP, Phillips SJ, O'Fallon WM, et al. Relationship of cardiac disease to stroke occurrence, recurrence, and mortality. Stroke 1992;23:1250-6.

10 Sandercock P, Bamford J, Dennis $M$, et al. Atrial fibrillation and stroke: prevalence in different types of stroke and influence on early and long term prognosis (Oxfordshire community stroke project). BMJ 1992;305:1460-5.

11 Anderson CS, Jamrozik KD, Broadhurst RJ, et al. Predicting survival for 1 year among different subtypes of stroke. Results from the Perth community stroke study. Stroke 1994;25:1935-44.

12 Tomita F, Kohya T, Sakurai M, et al; Hokkaido Atrial Fibrillation Study Group. Prevalence and clinical characteristics of patients with atrial fibrillation: analysis of 20,000 cases in Japan. Jpn Circ J 2000;64:653-8.

13 Bogousslavsky J, Van Melle G, Regli F. The Lausanne Stroke Registry: analysis of 1,000 consecutive patients with first stroke. Stroke 1988;19:1083-92.

14 Heinsius T, Bogousslavsky J, Van Melle G. Large infarcts in the middle cerebral artery territory. Etiology and outcome patterns. Neurology 1998;50:341-50.

15 Jorgensen HS, Nakayama H, Reith J, et al. Acute stroke with atrial fibrillation. The Copenhagen stroke study. Stroke 1996;27:1765-9.

16 Lowe GD, Jaap AJ, Forbes CD. Relation of atrial fibrillation and high haematocrit to mortality in acute stroke. Lancet 1983:1:784-6.

17 Kimura K, Kazui S, Minematsu K, Yamaguchi T, for the Japan Multicenter Stroke Investigators' Collaboration (J-MUSIC). Hospital-based prospective registration of acute ischemic stroke and transient ischemic attack in Japan. Journal of Stroke and Cerebrovascular Disease 2004;13:1-11.

18 Kimura K, Kazui S, Minematsu K, for the Japan Multicenter Stroke Investigator's Collaboration (J-MUSIC), et al. Analysis of 16,922 patients with acute ischemic stroke and TIA in Japan hospital-based prospective registration study. Cerebrovasc Dis 2004; 18:47-56.

19 van Swieten JC, Koudstaal PJ, Visser MC, et al. Interobserver agreement for the assessment of handicap in stroke patients. Stroke 1988;19:604-7.

20 Adams HP Jr, Davis PH, Leira EC, et al. Baseline NIH stroke scale score strongly predicts outcome after stroke: a report of the Trial of Org 10172 in Acute Stroke Treatment (TOAST). Neurology 1999;53:126-31.

21 Lavy S, Stern S, Melamed E, et al. Effect of chronic atrial fibrillation on regional cerebral blood flow. Stroke 1980;11:35-8.

22 Saxena $R$, Lewis $S$, Berge $E$, et al. Risk of early death and recurrent stroke and effect of heparin in 3169 patients with acute ischemic stroke and atrial fibrillation in the international stroke trial. Stroke 2001;32:2333-7.

23 Allen CMC. Predicting the outcome of acute stroke: a prognostic score. J Neurol Neurosurg Psychiatry 1984;47:475-80.

24 Wolf PA, Kannel WB, McGee DL, et al. Duration of atrial fibrillation and imminence of stroke: the Framingham study. Stroke 1983;14:664-7.

25 Censori B, Camerlingo M, Casto L, et al. Prognostic factors in first-ever stroke in the carotid artery territory seen within 6 hours after onset. Stroke 1993;24:532-5.

26 Kannel WB, Abbott RD, Savage DD, et al. Epidemiologic features of chronic atrial fibrillation: the Framingham study. N Engl J Med 1982;306:1018-22.

27 Feinberg WM, Blackshear JL, Laupacis A, et al. Prevalence, age distribution, and gender of patients with atrial fibrillation. Analysis and implications. Arch Intern Med 1995; 155:469-73.

28 Gustafsson C, Britton M. Pathogenetic mechanism of stroke in non-valvular atrial fibrillation: follow-up of stroke patients with and without atrial fibrillation. J Intern Med 1991;230:11-16.

29 Kaarisalo MM, Immonen-Raiha P, Marttila RJ, et al. Atrial fibrillation and stroke. Mortality and causes of death after the first acute ischemic stroke. Stroke 1997;28:311-15.

30 Lin HJ, Wolf PA, Kelly-Hayes $M$, et al. Stroke severity in atrial fibrillation. The Framingham study. Stroke 1996;27:1760-4.

31 Moroney JT, Bagiella E, Paik MC, et al. Risk factors for early recurrence after ischemic stroke: the role of stroke syndrome and subtype. Stroke 1998;29:2118-24.

32 Sage Jl, Van Uitert RL. Risk of recurrent stroke in patients with atrial fibrillation and non-valvular heart disease. Stroke 1983;14:537-40.

33 Chen ZM, Sandercock P, Pan HC, et al. Indications for early aspirin use in acute ischemic stroke: a combined analysis of 40000 randomized patients from the Chinese acute stroke trial and the international stroke trial. On behalf of the CAST and IST collaborative groups. Stroke 2000;31:1240-9. 